\title{
Barkhausen Noise Probes and Modelling: A Review
}

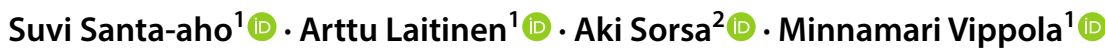

Received: 7 November 2018 / Accepted: 13 September 2019 / Published online: 21 September 2019

(c) The Author(s) 2019

\begin{abstract}
This review looks at the main types of magnetic Barkhausen noise (BN) probes that have been developed. The aim of this review is to summarize the existing knowledge of magnetic Barkhausen noise probes and the magnetic modelling of them. The BN probes have been the focus of many previous studies, but no sufficient review or conclusions have been made so far. This review focuses on combining information regarding the different types of $\mathrm{BN}$ probes and their modelling. The review is divided into two sections; in the first part the different designs and types of Barkhausen noise probes are introduced. The second part of the review deals with the BN probe modelling with various modelling software. Finally, a comparison of the experimental measurements is made and $\mathrm{BN}$ sensitivity is discussed.
\end{abstract}

Keywords FEM modelling $\cdot$ Magnetic Barkhausen noise $\cdot$ Non-destructive testing $\cdot$ Probes

\section{Introduction}

Barkhausen noise (BN) is a non-destructive magnetic measurement method, which has been used in many industrial applications for quality control and as an aid to verify process outcomes. The method is versatile because it is sensitive to changes in both stress state and microstructure. Huang and Qian [1] collected the latest advances in magnetic nondestructive testing and the application of this technique in their recent review article. The BN method has been used widely in grinding burn studies [2] of different ground (transmission) components and inspecting surface characteristics after different heat treatments [3]. Due to its stress sensitivity, $\mathrm{BN}$ is also used for detecting stresses in pipelines [4]. However, the $\mathrm{BN}$ method is not yet standardized; it is under development. Recently some guidelines for recommended practices have been published [2, 5]. Traditional BN measurement systems usually consist of a measurement device, electronics, computer and a sensor or probe consisting of a magnetization coil and pickup coil. In the literature, both terms "sensor" and "probe" are utilized to refer to the

Suvi Santa-aho

suvi.santa-aho@tuni.fi

1 Materials Science and Environmental Engineering, Tampere University, P.O. Box 589, 33014 Tampereen Yliopisto, Finland

2 Control Engineering, University of Oulu, Oulu, Finland magnetization and sensing unit for BN. In this paper, the term probe is used. As Durin and Zapperi [6] pointed out, there are no typical or standard designs, sizes and specifications for conventional BN probes. Deveci [7] stated that the variety of different possible probes for different applications is one of the main advantages of the $\mathrm{BN}$ method. In a simplified system for $\mathrm{BN}$ measurements, the magnetic circuit is formed by a copper coil wound on a core, which forms an electromagnet that is supplied by alternating current. The alternating magnetic field is then induced by the yoke to the studied surface. As White [8] summarizes, a large amplitude low frequency magnetization field is used to cycle the sample around the hysteresis loop. Cyclic magnetization is generated in the material, and the generated outcome of the irreversible domain wall motion is measured by the pickup coil as Barkhausen noise. The pickup coil detects the domain structure reconfigurations causing the changes in the magnetization as a response to the magnetization field [8]. The $\mathrm{BN}$ response expressed as voltage is induced in a pickup winding coil placed around the material sample or on its surface [9] according to Faraday's law of induction [4]. The electromagnetic field that is produced by the magnetizing yoke will decay exponentially into the depth in a direction perpendicular to the surface, due to eddy current damping. The electromagnetic skin depth will be affected by several material and measurement parameters, i.e., the frequency, conductivity and permeability of the material. The skin depth $\delta$ is given by the relation 
$\delta=\frac{1}{\sqrt{\pi f \sigma \mu_{o} \mu_{r}}}$,

where $\mathrm{f}$ is the frequency, $\sigma$ is the conductivity of the material, $\mu_{\mathrm{o}}$ is the permeability of vacuum and $\mu_{\mathrm{r}}$ is the relative permeability of the material [10].

Barkhausen noise is not produced uniformly throughout the magnetization cycle but is concentrated in two bursts of activity per cycle near the coercive field, whereas close to positive or negative hysteresis loop saturation the signal is minimal or zero [11]. This means that the $\mathrm{BN}$ probe needs to be fixed on the sample surface for at least half a magnetization cycle, when BN measurements are carried out. This traditional method of measuring Barkhausen noise can be considered a stationary BN technique. Rotating measurements are applied for components which are inspected by rotating the component under a stationary probe. For example, transmission components like crankshafts or camshafts can be inspected with either inline, automated or semi-automated systems [12]. Many versatile applications exist for $\mathrm{BN}$ measurements and, therefore, most applications require a customized probe design that accommodates the sample geometry. For flat surfaces with static measurements, the coupling between the electromagnetic yoke and the sample is similar in all cases and changes in the $\mathrm{BN}$ are due to variations in the sample's magnetic properties. Probe design is crucial for the operation of the whole measurement system. The choice of probe design parameters affect the generation of the magnetic fields that excite $\mathrm{BN}$ in the sample under study. In addition, the magnetic behaviour of the probe can be analysed by numerical simulation using the finite element method (FEM) to limit the need for experimental measurements.

\section{Barkhausen Noise Probes}

Traditionally, BN signals are measured with a probe or sensor, consisting of a magnetic excitation system, i.e., magnetizing yoke and pickup coil. The probe components are embedded normally in a case made of either aluminium [4] or stainless steel [7], where they are cast with epoxy inside the case [7]. To ensure the wear resistance of the probe, a wolfram carbide insert can be attached to the magnetizing pole pieces [7].

\subsection{Materials and Geometry: Magnetization Unit}

The magnetization unit can be placed in the same embedded case as the pickup unit or it can be external. The external magnetization unit can be used for example in a case of bearing measurements where the pickup is in a stationary location. As Moorthy [13] concluded, external magnetization can be carried out with an open loop solenoid or with a U-shaped or rectangular electromagnetic yoke with rounded areas in the upper parts of the magnetization legs. The C-shaped, U-shaped or rectangular yokes are more common and practical in industrial use than the solenoid. In the U-shape yoke, the copper coil can be wrapped in the upper area of the core. Prabhu Gaunkar et al. [14] studied the effect of core length on the magnetic field and concluded, based on their modelling, that the magnetic field strength decreases with increasing core length (path length from pickup to coil). Thus, in the magnetization unit, the copper coil for magnetizing has also been wrapped around both legs as demonstrated in Fig. 1a, based on Prabhu Gaunkar et al. [14-16], or wrapped around each of four magnetizing legs as in White's tetrapole probe [8]. However, for some special cases, for example in the magnetization of a wire sample, Capo-Sanchez et al. [17] reported the use of a solenoid with 700 turns of copper wire. Solenoid magnetization for a bar sample is demonstrated in Fig. 1b.

In some cases, a flux sensing coil or feedback coil for controlling the currents can also be placed in the magnetizing leg, as in the studies of White et al. [8, 18] and Vengrinovich and Tsukerman [19]. In White's assembly, the magnetizing coil was wrapped around the upper part (head bow) of the magnetization yoke and a flux sensing coil was wrapped at

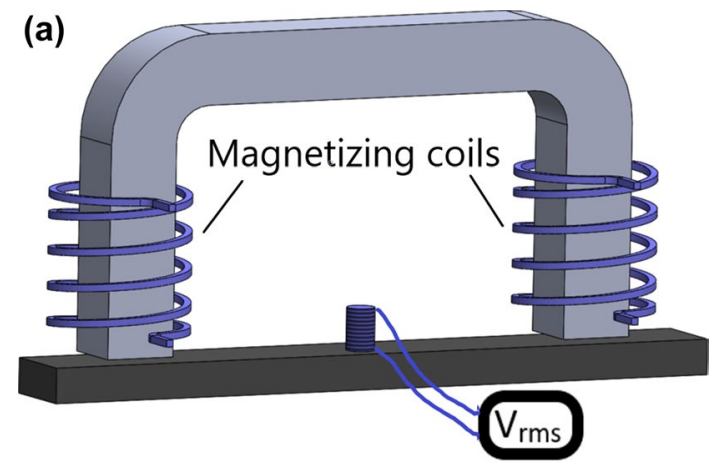

(b)

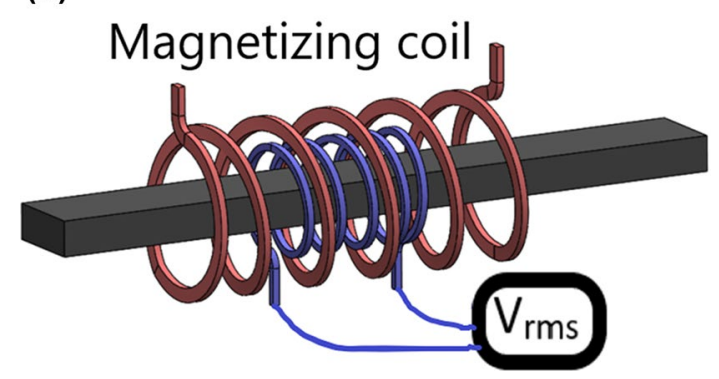

Fig. 1 Magnetization unit with a copper coil wrapped around both legs, schematic image based on [15] and $\mathbf{b}$ wrapped around the sample, schematic image based on [17] 
the bottom of the leg, as demonstrated in Fig. 2. This position for the flux sensing coil was justified as it best represents the flux entering and leaving the studied sample [18].

The single-head or single-core layout has been introduced in Lo et al. [20], for example. Stupakov [21] utilized a special single yoke measurement set-up where a C-core was used for the magnetizing yoke with the driving copper coil positioned on the head bow and two induction coils wrapped around the legs close to the sample. The induction coil recorded the magnetic flux in the head-sample magnetic circuit. Another single-core layout for special measurements was introduced by Kazanci et al. [22, 23], which was a miniaturized fixture for BN measurements for deep drilled holes. The material of the magnetizing yoke core and the pickup yoke core is usually ferrite, which is a ceramic homogeneous material composed of oxides, mainly iron oxide [24]. Ferrites are divided into two categories: $\mathrm{Mn}-\mathrm{Zn}$ ferrites and $\mathrm{Ni}-\mathrm{Zn}$ ferrites [24]. As Stupakov [21] concluded, the yoke core is usually chosen to be magnetically softer than the core of the sample in order to allow the magnetic field to penetrate easily and produce magnetization. In some special cases, a magnetizing yoke core made of iron has been studied $[13,14,25]$. The magnetization yoke in the studies of Moorthy [13] had a solid pure iron core. The permeability values depend on the composition and, in the case of iron, on the impurities, but generally ferrite has lower relative permeability values compared with iron [14]. Another important difference between the different core materials is the saturation magnetization value. A ferrite core yoke yields much lower saturation magnetization $(300 \mathrm{mT})$ than an iron core yoke [25]. The lower saturation magnetization of the ferrite core may be one reason for the differences in the observed pickup signal with different magnetizing yokes seen in the studies of Vértesy et al. [25]. Prabhu Gaunkar et al. [14] verified, by means of modelling, that the magnetic

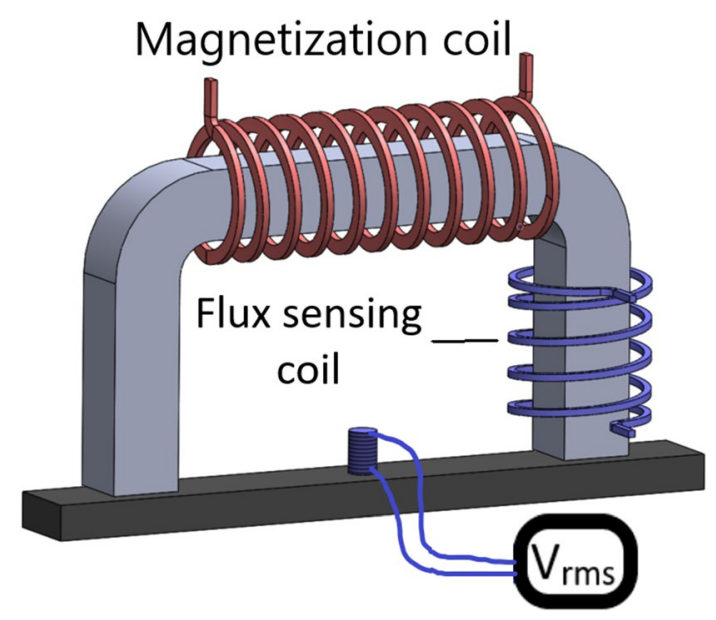

Fig. 2 Flux sensing coil attached to the magnetizing yoke. Schematic image based on [18] flux densities vary with different core materials. They [14] noticed that, among the materials that they studied, the 78 Permalloy had the highest and the iron core had the second highest magnetic flux density values. Due to the lower saturation magnetization of Permalloy Prabhu Gaunkar et al. [14] utilized the iron core instead to avoid saturation of the core material [14]. Stupakov has also used a core manufactured from laminated Fe-Si transformer steel [26]. Besides the above-mentioned materials used for the core, other alloys also exist e.g., Supermendur [8], with even higher saturation flux densities than iron or ferrites.

\subsection{Materials and Geometry: Pickup Unit}

For pickup units, the simplest form is an air coil where the copper wire is wound in a circular form. However, the air coil has relatively low sensitivity as explained in [27]. Thus, the addition of a soft magnetic ferrite core inside the copper coil leads to better sensitivity of the coil [27]. Prabhu Gaunkar et al. [15] verified with simulations that using a high magnetic permeability core material in the pickup enhances the recorded BN signal although the core material should not be saturated in the measurements. Pickup coil modifications have been studied by many researchers e.g., [17, 27]. For example, Stupakov et al. [28] tested many differently modified attached coil structures to observe their effect on the BN response. In their studies [28], two different pickup coil types were introduced: a pancake coil and a cylindrical coil wrapped around the sample, which are shown schematically in Fig. $3 \mathrm{a}$ and b. The pancake coil is also referred to as a pickup bobbin coil [26].

Stupakov et al. [28] utilized both an air core and a core made of CNS 12021 steel or Fe-Si transformer steel for the pancake coil. The cylindrical coil had a ferrite core of $25 \mathrm{~mm}$ in height. Capo Sanchez et al. [17] also studied a pancake pickup, which was wound around a small cylindrical plastic core with 200 turns. In addition, they [17] studied a pickup wound around the studied sample with 1000 turns of AWG44 copper wire. For the pickup yoke core, ferrite has been a widely used material. Stupakov et al. [26, 28] used laminated $\mathrm{Fe}-\mathrm{Si}$ transformer steel for the pickup core in their studies. In addition, Stupakov [26] used $\mathrm{Cu}$-shielding with a grounded case for the pickup. Prabhu Gaunkar et al. [15] used a nickel-zinc ferrite core for their pickup. Besides the use of different pickup core materials, Stupakov et al. [28] experimentally showed that the responses of different pickup coils with different materials were found to be fairly similar to each other. The main difference was with an air core: the addition of the core material to the air core pickup led to 3-5 times the amplification of the $\mathrm{BN}$ responses depending on the core material. The shielding with a copper-grounded probe case decreased the environmental noise compared with open ferrite coils [28]. 
(a)
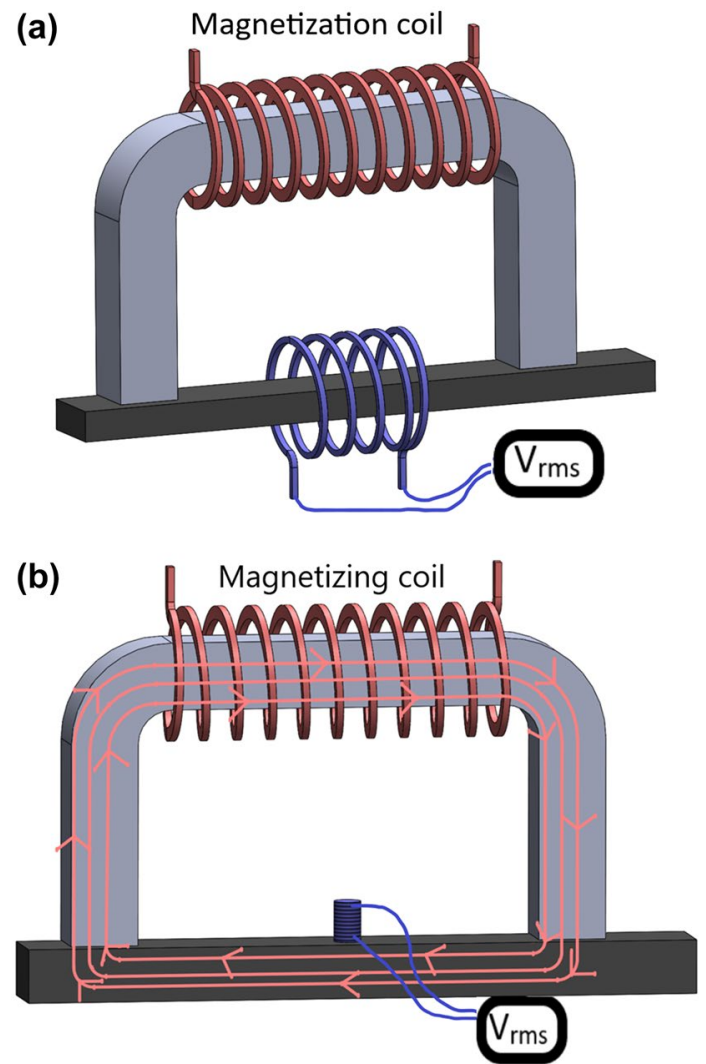

Fig. 3 a Cylindrical coil wrapped around the sample and b pickup pancake coil with core and copper winding on the sample surface. Schematic images based on [17]. The formed magnetic fields entering the sample are shown as magnetic flux arrows in (b)

\subsubsection{Pickup Placement}

Normally one pickup coil is located between the two magnetizing yoke feet as a separate unit. However, in some applications, a single magnetizing yoke is applied with multiple search pickup coils. In addition, Patel et al. [29] utilized two pickup coils to record $\mathrm{BN}$ : one coil to obtain flux density and one $\mathrm{H}$-coil to obtain the magnetic field, whereas the so-called tetrapole probes utilize two electromagnetic yokes and two pickups [19]. Augustyniak et al. [30] used a double-core set-up, where all four coils were wrapped on two perpendicular C-cores. In some specific geometries (rotational symmetry) the pickup coil can be wound around the sample as demonstrated in [8] or in [17, 28]. In this case, the flux is measured directly in the sample. However, for the typical BN measurements of nonrotationally symmetric samples, the optimal magnetic field sensing location would be on the sample surface in the region of interest.

\subsubsection{Probe Lift-Off and Other Geometrical Issues}

Typically probe design is application-specific as most applications require a customized probe design to accommodate the sample geometry. This means that the probe design takes the sample surface geometry into account, and the probe and its leg geometry is carefully considered to minimize the air gap at the surface. As White et al. [18] concluded, even small changes in the magnetizing unit yoke lift-off can produce large changes in magnetic circuit permeability, and thus the probe position dramatically affects the sensitivity of the Barkhausen effect. The air gap between the probe and the target specimen affects the magnetic coupling between the electromagnet's poles and the sample [8]. The air gap significantly reduces the permeability of the circuit formed by the core, sample and air gap, and also reduces flux density B [18]. The geometry of the magnetizing leg and pick-up surface depends on the geometry of the surface of the sample. For flat surfaces, flat-shaped pieces are normally used, while round-shaped ones are used for round surface samples [7]. In addition, the magnetizing pole pieces can be removable and attached so that they can be changed according to the surface to be measured [7]. However, Prabhu Gaunkar et al. [14] pointed out with their modelling studies that a curved magnetizing yoke core tip, with an appropriately calculated arc length, can ensure consistent magnetic flux coupling with varying surface geometries.

The pickup location with only minor lift-off can be verified by a spring-loaded pickup mechanism [26, 28, 31, 32].

The air gaps also have an effect on the pickup sensitivity and different pickups have been demonstrated to exhibit different sensitivity to air gaps or lift-offs. Stupakov et al. [28] noticed that a pickup with a soft magnetic core was more sensitive to air gaps than an FeSi or CSN 12021 steel core. Thus, ensuring the proper contact without air gaps is essential and spring loading should be considered. McNairnay [4] noticed that the lift-off effect scales with the coil diameter and increasing the size of the coil would ensure that the pickup assembly is less sensitive to the air gap or lift-off variations. White et al. [18] performed lift-off studies by placing plastic spacers between the sample and the probe. They [18] observed that the drive current must be increased with lift-off to compensate for the drop in magnetic circuit permeability due to the air gap.

\section{Commercial Probes}

Commercial Barkhausen noise system manufacturers offer readily available probes and measurement technology for Barkhausen noise inspections. Stresstech [33] produces probes, central units and readily available automated solutions for Barkhausen noise inspections for grinding burn 
studies, for example. Fraunhofer Institute IZFP (Germany) produces a 3MA device where Barkhausen noise can be measured along with other magnetic variables [34-36]. In addition, the Introscan system by Vengrinovich in Belarus is one Barkhausen noise related measurement system for stress qualitative assessment [19, 37]. The QASS company of Germany also manufactures a commercial Barkhausen noise inspection device, the QASS $\mu$ magnetic, mainly for hardness inspection utilizing known hardness references [38]. Stresstech is the biggest commercial manufacturer and its Barkhausen noise systems are utilized worldwide.

\section{Self-Made Probes}

\subsection{Self-Made Probes with Single Magnetizing Yoke}

For certain applications, commercial probes may have coupling or accessibility restrictions as White [8] mentioned when justifying his self-made probe manufactured for feeder pipe inspection. Barkhausen measurement is qualitative and the set-up may vary considerably for each laboratory, as it is not yet standardized [17]. For example, the magnetizing and pickup coils may have a different number of turns, different diameters of wire and different widths. Many researchers $[8,17,18,28,31,39-41]$ have built their own Barkhausen noise systems for research use. Commonly the U-shape or rectangular ferrite yoke with a winding copper coil is used for magnetization. Stupakov [26] states that this Barkhausen noise probe design is similar to the industrial and commercial probes provided by Stresstech and IZFP, in addition to other types of pickups. Therefore, the use of self-made probes allows simultaneous use of other sensors with the BN pickup coil such as Hall sensors and induction coils [28]. In many studies e.g., [16, 28, 39, 41], the Barkhausen noise search coil was equipped with Hall sensors. A vertical array of Hall sensors has been used to measure tangential magnetic surface fields at certain locations above the sample and field extrapolation technique used to determine the real sample magnetization [28], whereas an induction coil was utilized to control the sample magnetization [28].

\subsection{Double Core or Tetrapole Probes}

Besides the traditional single yoke and single pickup combinations for $\mathrm{BN}$ probes, various modifications have also been developed. A double-core measurement set-up called tetrapole was introduced by Vengrinovich and Tsukerman in 2004 [19]. The tetrapole probe includes two self-perpendicular electromagnets with pole pieces where the magnetic flux is created separately in each magnetizing coil [19]. The magnetic field is rotated in the surface plane of the sample and the linear superposition of two orthogonal magnetic fields is assumed. The flexible pickup is located in the middle of the coils. The use of tetrapole probes is justified in cases when there is anisotropy in the material as a tetrapole probe eliminates the need to rotate the probe between different measurement directions. Vengrinovich and Tsukerman have mainly studied stresses and created so-called directional diagrams (DD) of BN [19]. Augustyniak et al. [30] utilized a double-core magnetizing set-up, which used four coils that were wrapped around two perpendicular C-cores: called the $\mathrm{X}$-core and $\mathrm{Y}$-core referring to the measurement direction. The tetrapole BN probe has also been studied by Refs. $[4,8,42]$. White [42] introduced the tetrapole probe with either four cylindrical poles with magnetization and feedback coils on each pole or orthogonal U-cores with four legs with magnetization and feedback coils on each leg, depicted in Fig. $4 \mathrm{a}$ and b, respectively. The measurement system was adapted for BN anisotropy measurements [8]. FEM modelling showed that cores made with ferrite showed too low a saturation flux density and therefore a core made from high saturation laminated alloys (Supermendur) was used. [8]

\section{Surface Scanning Techniques and Moving Coil Probes}

\subsection{Continuous Magnetic Barkhausen Noise (CMBN)}

Conventional Barkhausen noise probes are usually used in the quality control of finished products or during their manufacture. However, traditional $\mathrm{BN}$ probes can be quite spaceand energy- consuming and thus, according to Hamfelt et al. [43], unsuitable for condition monitoring applications. New uses of Barkhausen noise in condition monitoring applications have generated a demand to change the conventional probe structure. The use of continuous magnetic Barkhausen noise (CMBN) measurements was first studied by Crouch [44], who utilized the concept of rotational permanent magnets for pipeline stress measurements. The magnet moving over the ferromagnetic sample produces a time-varying magnetic field that can excite $\mathrm{BN}$ if it is strong enough. The CMBN probe is an assembly of a magnet producing the magnetic field and a ferrite-cored coil for BN measurements [9]. Figure 5 shows a typical assembly of the CMBN device for measurements.

CMBN has been studied by many researchers $[9,11$, 45]. Continuous magnetic BN measurements can be made to detect anisotropy and the direction of the magnetic easy axis of ferromagnetic samples [45] as well as for studying the stress state [44]. Franco and Padovese [9] utilized the method to detect wall thickness loss and for the detection of plastic deformation of steel surfaces [11]. Franco Grijalba et al. [11] stated that the CMBN pickup coil has the same characteristics as a stationary BN pickup. The probe can be 
(a)
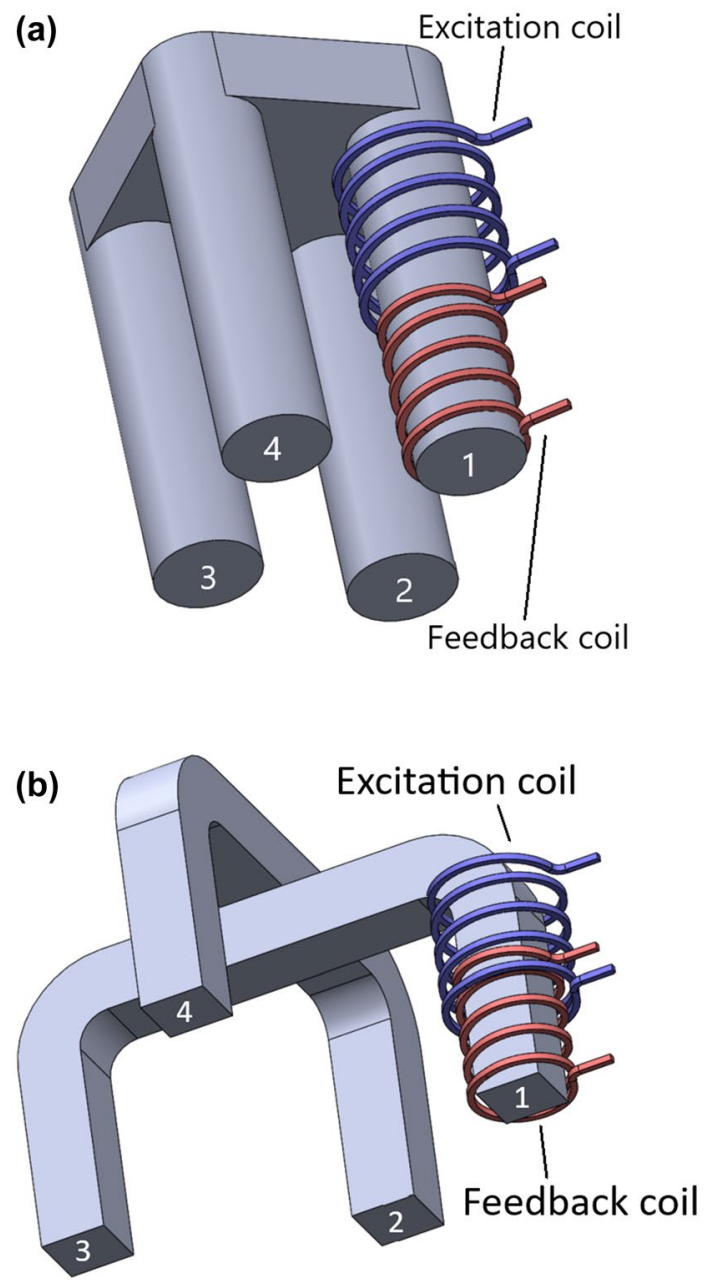

Fig. 4 Tetrapole probe with a four cylindrical poles and $\mathbf{b}$ orthogonal U-cores with magnetization and feedback coils. Schematic images based on [8]

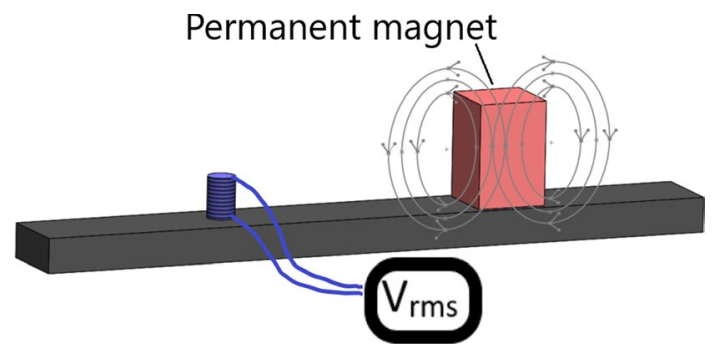

Fig. 5 Schematic image of a CMBN probe modified from [46]

fixed in a stationary position as the samples are moved with an XYZ table [9]. The magnet and the pickup need to be positioned in a way to maximize the magnetic field. Franco Grijalba et al. [11] found that the pickup coil behind the magnet provided increased Barkhausen noise signal values and greater sensitivity. The speed also has an influence on the measurements. Caldas-Morgan et al. [45] noticed that the $\mathrm{BN}$ generation was less noticeable at lower speeds. The same effect was noticed by Franco Grijalba et al. [46]. However, during scanning, the probe needs to be kept still for at least half of a magnetization cycle [9]. The CMBN measurement method is a relative technique and requires a calibration procedure [46].

\subsection{Permanent Magnet Probe}

Barkhausen noise has also been utilized in condition-based maintenance inspection for condition monitoring [43, 47]. Low power probe systems are required in the condition monitoring of fatigue damage for integrated bearings. Thus, a normal $\mathrm{BN}$ probe cannot be utilized as such and therefore, a $\mathrm{BN}$ probe was built with a permanent magnet that relies on the relative movement between the measured material and the probe. The BN probe consists of a solenoid coil, with or without a core, and a permanent magnet with magnetic flux parallel to the core of the coil. This kind of probe resembles a reluctance sensor with a non-varying reluctance circuit as stated by Hamfelt et al. [43].

\section{Utilization of Finite Element Modelling in Barkhausen Noise Probe Studies}

The finite element method (FEM) is a powerful engineering tool for all design, and includes research and development elements, such as building prototypes or optimizing parameters. This section is a summary of how different authors have utilized FEM in Barkhausen noise probe studies, and what kind of challenges have been experienced while doing so. Usually the general research question before the modelling is how the selection of the probe design parameters will affect the generation of magnetic fields used to excite $\mathrm{BN}$ in specimens, as stated by Prabhu Gaunkar et al. [14].

Without FEM, no information can be obtained about the Barkhausen noise probe, such as the strength of the generated magnetic field. Even though this is vital information, it cannot be measured from inside the material. By using FEM, several design problems can be solved, i.e., how changing the geometry and materials or how changing the input parameters affect the magnetic flux field. The modelling of the $\mathrm{BN}$ probe allows many different types of variations to be used in studying the magnetic flux densities and creation of the magnetic field. Typical variables in the probe assembly that can be modelled are the air gap or lift-off [40, 48], the shape of the yoke [40, 49], the number of turns of the magnetization coil [11], the shape of the pickup coil and the number of turns of the pickup coil [11].

In order to obtain usable results from simulations, a great deal of pre-existing information about the magnetizing 
parameters of the BN probe is required. In the FEM software ANSYS, the coil is modelled as a uniform density load domain with a value of $\mathrm{A} / \mathrm{m}^{2}$. In COMSOL software, this can be done by modelling the coil as a domain with the number of coil turns, coil diameter and input current/ voltage. The coil is then analysed with the Coil Geometry Analysis tool in COMSOL. In this step, the coil operation is studied in the simulations. The non-linear behaviour of the materials is required to ensure sufficient accuracy of the results in simulations that involve magnetic saturation. This can be taken into account with both ANSYS and COMSOL. In most publications, two commercial finite element programs: COMSOL Multiphysics and ANSYS were used, e.g., Franco et al. [9], Augustinyak et al. [29], Garstka and Stefanik [40], Laitinen et al. [50], Hao et al. [51], Persson et al. [52]. In addition, the Opera 3D program has been utilized in Barkhausen noise probe design [53].

\subsection{COMSOL Multiphysics}

Hao et al. [51, 54] used COMSOL Multiphysics in 2D for calculating the effective permeability of an actual two-phase microstructure containing ferrite and austenite. Their experimental studies concentrated on detecting the ferrite ratio of dual-phase steels [51] and detecting decarburization [54] from the probe outputs. In [51], the effective permeability was calculated with different ferrite fractions and the results were compared to the probe response. The results were heavily affected by the shape and distribution of the ferrite domains. The first step was to model the effective relative permeability for the ferrite-austenite microstructure; the second step was to link the effective relative permeability changing with the ferrite fraction to the probe output, using a finite element probe output model with the particular probe geometry. In this way, different microstructures containing different amounts of ferrite and probe designs can be considered separately or in combination. Zhou et al. [55] continued a similar type of modelling work as Hao et al. [51] with COMSOL, extending the modelling to 3D and adding 2nd phase distribution relative permeability calculations to the model of ferrite-austenite phase mixtures. It was important to study the 3D aspect in the modelling because the probe interacts with the microstructure in three dimensions.

Prabhu Gaunkar et al. [14] studied the different magnetizing core tip curvature, core length and effects of core materials using the AC/DC module of COMSOL Multiphysics. Persson et al. [52] modelled a soft iron C-core with a magnetizing coil and a layered steel plate with COMSOL. The layers represented different steel phases, such as martensite, bainite and pearlite. The difference between the steel phases was made by assigning a relative permeability value resembling their real-life counterparts to each material. The current authors, Laitinen et al. [50], used the commercial COMSOL software with the AC/DC module for modelling the magnetization of the pickup coil with several assembly configurations, such as an E-core and a pot core.

\subsection{ANSYS}

Augustinyak et al. [30] studied the magnetization of a double core set-up using ANSYS, which included tetrapole magnetizing coils with time-dependent density loading. Pal'a et al. [49] studied the effect of an air gap from 50 to $200 \mu \mathrm{m}$ between the probe and the sample, investigating the reproducibility of the measuring conditions. Pal'a et al. [49] also modelled different shapes of magnetizing yoke (plane or cylindrical) in 2D with ANSYS and in 3D with FEM. It was noticed that cylindrically shaped legs required higher magnetization currents compared with plane legs to gain the same tangential magnetic field generation. The cylindrical shape for yoke legs is only valid for stabilizing the magnetic field in a sample with large air gaps and large magnetizing currents. Garstka and Stefanik [40] studied the effect of changing the geometry of the magnetization yoke on the distribution intensity of the magnetic field using ANSYS. The electromagnetic calculations were based on the fundamental Maxwell field equations, and on Biot-Savart and Ampere's laws. They [40] studied square, rounded and concave profiled pole shoes. In addition, they also studied a case of how adding an air gap between the yoke and rounded sample changed the magnetic flux. The most favourable case, with the most uniform distribution of flux density and flux lines having the smallest relative gradient for magnetization, was obtained utilizing concave profiled pole shoes on rounded tubes $(\mathrm{B}=1.5 \mathrm{~T})$. The air gap gave the worst results; it decreased the value of $B$ drastically at the surface $(B=50 \mathrm{mT})$. In this case, the most uniform contact with the sampled surface proved the most favourable case. Franco and Padovese [9] utilized ANSYS FEM in deciding the optimal placement for the pickup coil in a set-up featuring a permanent magnet. The simulation also took the non-linear magnetic behaviour of the materials into account.

\subsection{Opera 3D}

Laukkanen [53] performed comparisons between the probe model and measurements to verify the model. The model was created with a commercial program called Opera 3D from Vector Fields with an ELEKTRA steady-state solver.

\section{Barkhausen Noise Pickup Modelling and Experimental Studies}

Most modelling studies concentrate on the BN magnetizing coil alone [30, 40, 51-53]. The modelling of magnetic fields is challenging as magnetizing coil modelling results do not 
reveal anything about the actual Barkhausen noise signal that comes from the sample to the pickup coil-it only provides information about the effect of the magnetization field on the pickup coil. However, it would be more important to gain information about how the pickup coil operates and its sensing capabilities. This is a hard task to achieve with modelling and thus a reverse approach might be needed: studying the pickup coil magnetization ability. The study [50] carried out by the current authors focused on pickup modelling. This approach was more valid for probe research and development design. It should be noted that pickup coil magnetization calculations only give the theoretical magnetic flux strength created by the pickup coil, from which suggestions about the strength and quality of the BN signal can be made. They are not directly proportional to each other and thus should always be verified by actual measurements.

Franco Grijalba et al. [11] have concentrated on continuous magnetic Barkhausen noise (CMBN) pickup coils. In their experimental studies, the CMBN pickup coil parameters (number of turns, wire diameter and coil height) were changed. The coil having the highest number of turns and the thickest wire provided larger amplitude signals than the coil with a smaller number of turns with the thinnest wire, although the result for flaw capability separation was worse with the coil having the highest number of turns. Prabhu Gaunkar et al. [15] studied the effect of different sample material permeabilities on pickup coil magnetization and came to the conclusion that the pickup core permeability should be high to have the best sensitivity, but the effects of mutual inductance between the magnetizing coil and the sensing coil also need to be taken into consideration.

The main aim of the studies by McNairnay [4] and White [8] was to achieve the smallest sensing radius of the pickup coil to perform the most accurate sensing. The small sensing radius was performed by employing a ferrite core inside a coil which was inside a sheath and copper shield. This probe structure was called a pot core. Deveci [7] also studied this pot core probe design and its effect on experimental measurements based on White's [8] studies. The pot core probe in [7] had a new design, which consisted of a brass shield as the outermost layer, a ferrite core as the inner layer with a sheath cylinder made of ferrite between them. The sheath was believed to focus the sensing pickup area into a smaller area to gain better signal outcome. The result was that the pot core did not work significantly better than the traditional probe design without the shield and sheath.

Laitinen et al. [50] studied the effect of a similar kind of pot core pickup with a brass shield and compared the outcome of the experimental measurements with the standard pickup. Both pickups were used with external magnetization to study two hydrogen burn marks on a bearing. Based on the modelling results, the pot core pickup with brass shielding would have gained higher magnetization located in a wider area than the standard pickup. In the experimental measurements, the pot core with brass shielding gave a better spatial resolution of the burn marks than the standard pickup. The signal with the pot core pickup was much more concentrated and gave a smaller peak width for the shallow burn marks than with the standard pickup. Therefore, based on the studies of Deveci [7] and Laitinen et al. [50], it can be said that the pot core pickup works on a case-by-case basis. Deveci [7] used the magnetization located inside the same probe whereas Laitinen et al. [50] utilized external magnetization. Laitinen et al. [50] used COMSOL to study the magnetization plots of a standard pickup, pot core pickup and e-core pickup, which is a modification of the pot core where a ferrite core is connected to a ferrite sheath from the top with a connecting ferrite bridge. The modelling results showed that this ferrite bridge would increase the magnetizing effect on the surface of the studied steel. The difference between the pot core and e-core is the solid ferrite body directing the magnetic field flux into the component. The addition of copper shielding showed a decrease in magnetic flux densities in the surface for all pickup configurations.

\section{Sensitivity of a BN Probe}

Signal sensitivity can be said to be a result of the relationship between the applied input and the output signal. Magnetization of the studied sample creates BN events for the pickup coil to detect. If magnetization is not suitable or there are air gaps [18], naturally the possibility to record the BN events is also decreased. Thus, the pickup also plays an important role. The whole accuracy of the measurements depends on the accuracy of both the calibration and the measurement themselves, as stated by Vengrinovich et al. [56]. The repeatability of $\mathrm{BN}$ measurements might be challenging because the magnetic fields cannot be measured directly within the material [30]. The detected BN signal also depends on the stress-state, microstructural inhomogeneity, magnetizing field produced by the magnetizing coils, core geometry, probe-to-specimen coupling and spacing between the core tips of the sample, as Prabhu Gaunkar et al. [14] concluded. Therefore, the optimization of the probe configuration would improve the sensitivity, reproducibility and accuracy of the detected Barkhausen signals, as stated in [14]. In addition, the probe materials need to be carefully selected as pointed out by [15]. The most suitable configuration is case-dependent and should be selected based on the application and its requirements. For example, the use of tetrapole probes is justified in cases when there is anisotropy in the material as a tetrapole probe eliminates the need to rotate the probe between different measurement directions, whereas selfmade probes with variable coil turns allow more detailed sensitivity adjustment. 
In modelling, the sensitivity of the probe was observed to be improved when a high-permeability core material with high saturation magnetization was utilized in the pickup coil with a large number of turns. As Tumanski [27] pointed out in his review, it is widely known and can be seen from the equation of Faraday's law that a high coil sensitivity can be obtained by using a large number of turns (n) and large active area (A). He [27] also stated that the optimization process for coil performance is no easy task. To make the coil probe with a ferromagnetic core more sensitive, the length of the core (or rather the ratio $\frac{l}{D_{i}}$ where 1 is the length and $D_{i}$ is the inner diameter of the coil), should be made as large as possible, since the sensitivity is proportional to $1^{3}$ [27]. Thus, in the case of a high-permeability material, the sensitivity of the probe depends mostly on the geometry of the core [27]. Capo Sanchez et al. [17] carried out a comparison of different $\mathrm{BN}$ probe configurations with measurements and found the magnetizing yoke-pancake pickup combination to be the most sensitive, which is also the most commonly utilized assembly among commercial probes.

In the studies of Blaow and Shaw [57], it was established that the number of turns in the detection coil is an important parameter that controls the shape of the BN amplitude peaks. The sensitivity of the two-peak BN profile characteristics was also found to depend on the magnetization field strength. In a low magnetizing field, the BN peak height was observed to increase as the number of turns of the detection coil increased. Therefore, based on the $\mathrm{BN}$ profile when increasing the number of pickup coil turns, Blaow and Shaw concluded that the detection depth could be enhanced by the number of turns of the pickup coil [57]. In the case of continuous magnetic $\mathrm{BN}$, the position of the probe and the magnet during motion was found to be an important factor for optimization of measurement sensitivity. The scanning speed and positioning of the probe need to be set in a way to maximize the magnetic field because $\mathrm{BN}$ generation is related to the magnetic field intensity [9].

\section{Conclusion}

In this paper, a review of the advances in the range of $\mathrm{BN}$ probes and modelling was presented. The $\mathrm{BN}$ probe has been studied but no thorough review or conclusions have been made so far. Thus, this review focused on combining the information regarding different types of $\mathrm{BN}$ probes and their modelling. The detailed comparison of different $\mathrm{BN}$ probes is challenging because they all seem to be built differently with different materials. Also, the materials, geometry and treatments in the studied samples vary and are not the same in the separate studies. BN measurements will only be consistent and comparable in cases where the magnetization distribution is achieved similarly in each measurement, and the circuit flux density control is an effective means for achieving this goal, as stated by White [18]. It only remains to be said that, besides basic studies, there is still a need to obtain better understanding of how various factors influence the measurement system. Optimizing the performance of probes for $\mathrm{BN}$ measurements can be carried out by means of probe modelling. Modelling with FEM will be utilized more in future to build probes more effectively. With FEM, changes in the probe design and materials can be easily modified to study their influence. The future trend of the utilization of FEM might be in modelling the actual microstructures of the studied samples to predict the magnetic field flux occurrence and to perhaps modify the probe materials based on the studied sample material. This assumption is based on the FEM microstructure model developed by Hao et al. [50] to predict relative permeability based on actual microstructures.

The challenges most authors face during magnetic field simulations are related to magnetic saturation, which requires a more refined mesh in the areas of saturation, and some optimization to converge on a solution. Finding the solution is often a simulation based on trial-and-error, which is highly time-consuming. It is also worth noting that there has not been any approach for modelling the Barkhausen noise phenomenon itself. This is due to the complex nature of the process, such as creating the magnetic domains for the material, which may not be possible with the current simulation tools. At present, if we wish to study the BN phenomenon itself, the approach of Hao et al. [51] and Zhou et al. [55] linking the effective permeability of the microstructure to the probe output appears to be the most feasible.

Acknowledgements Mika Kiviluoma, B.Sc., research assistant is thanked for his help with SolidWorks. The financial support from the Academy of Finland is gratefully acknowledged.

Open Access This article is distributed under the terms of the Creative Commons Attribution 4.0 International License (http://creativeco mmons.org/licenses/by/4.0/), which permits unrestricted use, distribution, and reproduction in any medium, provided you give appropriate credit to the original author(s) and the source, provide a link to the Creative Commons license, and indicate if changes were made.

\section{References}

1. Huang, H., Qian, Z.: Recent advances in magnetic non-destructive testing and the application of this technique to remanufacturing. Insight 60(8), 451-462 (2018)

2. Barkhausen Noise Inspection for Detecting Grinding Burns in High Strength Steel Parts (2018) SAE International, Aerospace Recommended Practice, ARP4462

3. Holmberg, J., Lundin, P., Olavison, J., Sevim, S.: Non-destructive testing of surface characteristics after nitrocarburizing of three different steel grades. In: 12th International Conference on 
Barkhausen Noise and Micromagnetic Testing (ICBM12), 25-26 Sept 2017, Dresden (2017)

4. McNairnay, P.: Magnetic Barkhausen Noise Measurements Using Tetrapole Probe Designs, Master's Thesis, Queen's University, Kingston (2014)

5. Tomkovski, R. (ed.): The Barkhausen Noise Measurement Good Practice Guide. KTH Royal Institute of Technology, Stockholm (2018)

6. Durin, G., Zapperi, S.: The Barkhausen effect. In: Bertotti, G., Mauergoyz, I. (eds.) The Science of Hysteresis, vol. I-III, pp. 181-267. Elsevier, Amsterdam (2005)

7. Deveci, M.: Nondestructive Determination of Case Depth by Barkhausen Noise method, M.Sc. Thesis, Tampere University of Technology, Tampere (2016)

8. White, S.A.: A Barkhausen Noise Testing System for CANDU® Feeder Pipes, Ph.D. Thesis, Queen's University, Kingston (2009)

9. Franco, F.A., Padovese, L.R.: NDT flaw mapping of steel surfaces by continuous magnetic Barkhausen noise: volumetric flaw detection case. NDT\&E Int. 42, 721-729 (2009). https://doi. org/10.1016/j.ndteint.2009.06.007

10. Chikazumi, S.: Physics of Magnetism. Wiley, New York (1964)

11. Franco Grijalba, F.A., Padovese, L.R.: Non-destructive Flaw mapping of steel surfaces by the continuous magnetic Barkhausen noise method: detection of plastic deformation. J. Nondestruct. Eval. 37, 26 (2018). https://doi.org/10.1007/s10921-018-0480-6

12. https://www.stresstech.com/en-fi/products/barkhausen-noise -equipment/for-crankshafts/. Accessed 7 Nov 2018

13. Moorthy, V.: Important factors influencing the magnetic Barkhausen noise profile. IEEE Trans. Magn. 52(4), 1-13 (2016). https://doi.org/10.1109/TMAG.2015.2502222

14. Prabhu Gaunkar, N., Kypris, O., Nlebedim, I.C., Jiles, D.C.: Optimization of sensor design for Barkhausen noise measurement using finite element analysis. J. Appl. Phys. 115(1-4), 17E512 (2014). https://doi.org/10.1063/1.4864438

15. Prabhu Gaunkar, N., Nlebedim, I., Jiles, D.C.: Approach for improving the sensitivity of Barkhausen noise sensors with applications to magnetic nondestructive testing. Mater. Eval. 10, 1377-1383 (2015)

16. Stupakov, A., Perevertov, O., Zablotskii, V.: A system for controllable magnetic measurements of hysteresis and Barkhausen noise. IEEE Trans. Instrum. Meas. 65(5), 1087-1097 (2016)

17. Capó Sánchez, J., de Campos, J.M., Padovese, L.R.: Comparison between different experimental set-ups for measuring the magnetic Barkhausen noise in a deformed 1050 steel. J. Nondestruct. Eval. 36, 66 (2017)

18. White, S., Krause, T., Clapham, L.: Control of flux in magnetic circuits for Barkhausen noise measurements. Meas. Sci. Technol. 18, 3501-3510 (2007). https://doi.org/10.1088/0957-0233/18/11/034

19. Vengrinovich, V., Tsukerman, V.: Stress and texture measurement using Barkhausen noise and angular scanning of driving magnetic field. In: 16th World Conference on Nondestructive Testing (16th WCNDT), Aug 30-Sept 3 2004, Montreal (2005)

20. Lo, C.C.H., Paulse, J.A., Jiles, D.C.: A magnetic imaging system for evaluation of material conditions using magnetoresistive devices. IEEE Trans. Magn. 39(5), 3453-3455 (2003)

21. Stupakov, O.: Investigation of applicability of extrapolation method for sample field determination in single-yoke measuring setup. J. Magn. Magn. Mater. 302(2), 279-287 (2006)

22. Kazanci, E., Bekar, T., Iyiuyarlar, B., Pihava, E.G., Bicim, B.: Investigation of impact of autofrettage operation on thick walled steel tube. In: 12th International Conference on Barkhausen Noise and Micromagnetic Testing (ICBM12), 25-26 Sept 2017, Dresden (2017)

23. Kazanci, E., Iyiuyarlar, B., Pihava, E.G., Bicim, B.: Investigation of surface integrity of deep hole drilling of thick walled steel tube. In: 12th International Conference on Barkhausen
Noise and Micromagnetic Testing (ICBM12), 25-26 Sept 2017, Dresden (2017)

24. McLyman, M.T.: Transformer and Inductor Design Handbook. Revised and Expanded, 3rd edn. Marcel Dekker, New York (2004)

25. Vértesy, G., Uchimoto, T., Takagi, T., Tomas, I.: Magnetic adaptive testing: influence of experimental conditions. In: Takahashi, S., Kikuchi, H. (eds.) Electromagnetic Nondestructive Evaluation (X). IOS Press, Amsterdam (2007)

26. Stupakov, O.: Stabilization of Barkhausen noise readings by controlling a surface field waveform. Meas. Sci. Technol. (2014). https://doi.org/10.1088/0957-0233/25/1/015604

27. Tumanski, S.: Induction coil sensors-a review. Meas. Sci. Technol. 8, R31-R46 (2007). https://doi.org/10.1088/0957-0233/18/3/ R01

28. Stupakov, O., Palá, J., Takagi, T., Uchimoto, T.: Governing conditions of repeatable Barkhausen noise response. J. Magn. Magn. Mater. 321, 2956-2962 (2009)

29. Patel, H.V., Zurek, S., Meydan, T., Jiles, D.C., Li, L.: A new adaptive automated feedback system for Barkhausen signal measurement. Sens. Actuator A 129, 112-117 (2006)

30. Augustyniak, M., Augustyniak, B., Piotrowski, L., Chmielewski, M.: Determination of magnetisation conditions in a double-core Barkhausen noise measurement set-up. J. Nondestruct. Eval. 34, 16 (2015). https://doi.org/10.1007/s10921-015-0288-6

31. Augustyniak, B., Piotrowski, L., Chmielewski, M., Kosmas, K., Hristoforou, E.: Barkhausen noise properties measured by different methods for deformed Armco samples. IEEE Trans. Magn. 46(2), 544-547 (2010)

32. Yardley, V.A.: Magnetic Detection of Microstructure Change in Power Plant Steels, Ph.D. Thesis, University of Cambridge, UK, https://www.repository.cam.ac.uk/bitstream/handle/1810/22182 5/Yardley_thesis.pdf. Accessed 7 Nov 2018 (2003)

33. www.stresstech.com. Accessed 3 Aug 2018

34. Gabi, Y., Wolter, B., Kern, R., Conrad, C., Gerbershangen, A.: Simulation of electromagnetic inspection techniques using FEM analysis. In: 19th World Conference on Non-destructive Testing, 13-17 June 2016, Munich (2016)

35. Wolter, B., Szielasko, K., Tschuncky, R., Christian, C.: Micromagnetic testing at Fraunhofer IZFP: highlights and experiences of more than 3 decades of research. In: 12th International Conference on Barkhausen Noise and Micromagnetic Testing (ICBM12), 25-26 Sept 2017, Dresden (2017)

36. Tschuncky, E., Szielasko, K., Altpeter, I.: Hybrid Methods for Materials Characterization, Materials Characterization Using Nondestructive Evaluation (NDE) Methods, pp. 263-291. Woodhead Publishing, Sawstonm (2016). https://doi.org/10.1016/B9780-08-100040-3.00009-2

37. http://iaph.bas-net.by/VDev/Introscan/introscane.html. Accessed 3 Aug 2018

38. http://www.qass.net/wp/products/qass-\%C2\%B5magnetic-hardn ess-testing/?lang=en. Accessed 3 Aug 2018

39. Xiucheng, X., Ruihuan, Z., Bin, W., Cunfu, H.: Quantitative prediction of surface hardness in $12 \mathrm{CrMoV}$ steel plate based on magnetic Barkhausen noise and tangential magnetic field measurements. J. Nondestr. Eval. 37, 38 (2018). https://doi.org/10.1007/ s10921-018-0486-0

40. Garstka, T., Stefanik, A.: Modelling of the magnetic field in Barkhausen measurement method. In: 12th Scientific International Conference, Achievements in Mechanical and Materials Engineering AMME 2003, Gliwice-Zakopane, 7-10 Dec 2003, pp. 101-104 (2004)

41. Lasaosa, A., Gurruchaga, K., Garcia Navas, G., Martinez-deGuerenu, A.: Characterisation of in-depth stress state by magnetic Barkhausen noise on machined steel acquiring different frequency bands. Adv. Mater. Res. 996, 373-379 (2014) 
42. White, S., Krause, T.W., Clapham, L.: A multichannel magnetic flux controller for periodic magnetizing conditions. IEEE Trans. Instrum. Meas. 61(7), 1896-1907 (2012)

43. Hamfelt, J., Gustafsson, J., van Deventer, J., Löfqvist, T., Häggström, F., Delsing, J.: A passive Barkhausen noise sensor for low-power application. In: IEEE International instrumentation and Measurement Technology Conference (I2MTC), 23-26 May 2016, Taipei. https://doi.org/10.1109/I2MTC.2016.7520374 (2016)

44. Crouch, A.E., Beuker, T.: In-line stress measurement by the continuous Barkhausen method. In: IPC2004 International Pipeline Conference, 4-8 Oct 2004, Calgary (2004)

45. Caldas-Morgan, M., Padovese, L.R.: Fast detection of the magnetic easy axis on steel sheet using the continuous rotational Barkhausen method. NDT\&E Int. 45, 148-155 (2012)

46. Franco Grijalba, F.A., Padovese, L.R.: Non-destructive scanning for applied stress by the continuous magnetic Barkhausen noise method. J. Magn. Magn. Mater. 446, 231-238 (2018)

47. Hamfelt, J.: Evaluation of a New Barkhausen Noise Sensor, Master's Thesis, Luleå University of Technology, Luleå (2015)

48. Katoh, M., Nishio, K., Yamaguchi, T.: FEM study on the influence of air gap and specimen thickness on the detectability of flaw in the yoke method. NDT\&E Int. 33, 333-339 (2000)

49. Pala, J., Bydžovský, J., Stupakov, O., Tomáš, I., Kadlecová, J., Jančárik, V.: Influence of yoke legs shape on air gap uncertainty. J. Electr. Eng. 57(8), 100-104 (2006)

50. Laitinen, A., Santa-aho, S., Lukinmaa, H., Suominen, L., Vippola, M.: Barkhausen noise Potcore sensor simulations with COMSOL. In: 12th International Conference on Barkhausen Noise and Micromagnetic Testing (ICBM12), 25-26 Sept 2017, Dresden (2017)

51. Hao, H.J., Yin, W., Strangwood, M., Peyton, A.J., Morris, P.J., Davis, C.J.: Modelling the electromagnetic response of two-phase steel microstructures. NDT\&E Int. 43, 305-315 (2010)
52. Persson, G.: On the modelling of a Barkhausen noise sensor. In: 12th European Conference on Non-destructive Testing (12th ECNDT), 11-15 Jun 2018, Gothenburg (2018)

53. Laukkanen, M.: Magnetic field simulations of a Barkhausen noise sensor, Master's Thesis, University of Jyväskylä, Finland (2005)

54. Hao, X.J., Yin, W., Strangwood, M., Peyton, A.J., Morris, P.J., Davis, C.J.: Characterization of decarburization of steels using a multifrequency electromagnetic sensor: experiment and modeling. Metall. Mater. Trans. A 40, 745-756 (2009)

55. Zhou, L., Davis, C., Kok, P., Van Der Berg, F.: Magnetic NDT for steel microstructure characterisation: modelling the effect of second phase distribution on magnetic relative permeability. In: 19th World Conference on Non-destructive Testing, 13-17 June 2016, Munich (2016)

56. Vengrinovich, V., Tsukerman, V., Denkevich, Y. Barkhausen effect based sensor and intellectual system for accurate stress measurements and monitoring in harsh environmental conditions. In: 15th World Conference on Nondestructive Testing, 15-21 October 2000, Rome (2000)

57. Blaow, M.M., Shaw, B.A.: Magnetic Barkhausen noise profile analysis: effect of excitation field strength and detection coil sensitivity in case carburized steel. Mater. Sci. Appl. 5, 258-266 (2014). https://doi.org/10.4236/msa.2014.55030

Publisher's Note Springer Nature remains neutral with regard to jurisdictional claims in published maps and institutional affiliations. 